\title{
Beam Profile Measurements on RHIC ${ }^{\star}$
}

\author{
R. Connolly, R. Michnoff, T. Moore, T. Shea, and S. Tepikian \\ Brookhaven National Laboratory, Upton, NY 11973
}

\begin{abstract}
The Relativistic Heavy Ion Collider (RHIC) at Brookhaven National Lab was commissioned during the summer of 1999. Transverse beam profiles on RHIC are measured with ionization profile monitors (IPM s). An IPM measures beam profiles by collecting the electrons liberated by residual gas ionization by the beam. The detector is placed in the gap of a dipole magnet to force the electrons to travel in straight lines from the beamline center to the collector. One IPM was tested and it measured the profiles of a single gold bunch containing $10^{8}$ ions on consecutive turns. We show an example of one of these profiles giving transverse emittance. Also several profiles are combined into a mountain-range plot which shows betatron oscillations at injection.
\end{abstract}

\section{INTRODUCTION}

The Relativistic Heavy Ion Collider (RHIC) at Brookhaven National Laboratory is a pair of concentric synchrotrons in which counter-rotating beams intersect at six points [1]. Beams ranging in mass from protons $\left(E_{\max }=250 \mathrm{GeV}\right)$ to fully-stripped gold $\left(E_{\max }=100 \mathrm{GeV} /\right.$ nucleon) are accelerated and stored for several hours. There are detectors at four of the six intersection points.

Transverse beam profiles are measured with ionization profile monitors (IPM s)[2,3]. An IPM collects the electrons in the beamline resulting from residual gas ionization during a bunch passage. The electrons are swept transversely from the beamline and collected on strip anodes oriented parallel to the beam axis. As each bunch passes through the detector, the charge pulses are amplified, integrated, and digitized to give the bunch profile. Four IPM $s$ are installed to measure vertical and horizontal profiles in the two rings.

The beam profile is measured primarily to find the transverse beam emittance [4]. The beam radius at the IPM is equal to the square root of the betatron function, $\beta_{\text {IPM, }}$, times the emittance. The value of $\beta_{\mathrm{IPM}}$ is either calculated or measured and the beam radius is determined by profile measurements.

This paper describes the beam profile detector used on RHIC and shows data collected during the RHIC commissioning experiments. A single-bunch profile is shown from which we calculate the beam emittance. Also several profiles made on consecutive turns are assembled into a mountain-range plot which shows betatron oscillations at injection.

*Work performed under the auspices of the U.S. Department of Energy. 


\section{DETECTOR DESIGN}

RHIC will accelerate and store many different beams and the IPM s are located $50 \mathrm{~m}$ from an intersection point where the lattice optics varies with the requirements of the experiment. Depending on energy, ion species and optics the rms width of the beam can vary from $0.7 \mathrm{~mm}$ to $6.5 \mathrm{~mm}$. The channel spacing has to be small enough that a reasonable measurement can be made on the most narrow beam but the collector has to be wide enough to see the largest beam with some allowance for missteering and betatron oscillations.

The compromise chosen was to use 64 channels spaced $0.6 \mathrm{~mm}$ apart. Only $6 \sigma$ of the widest beam fits on the collector and $6 \sigma$ of the narrowest beam fits on only 7 channels. However the measurement of a Gaussian beam of $\sigma=0.7 \mathrm{~mm}$ with a collector $0.6 \mathrm{~mm}$ wide yields a rms width less than $4 \%$ larger than the true width.

The signal is the electrons released by ionizing collisions of the beam with the residual gas. Since RHIC has superconducting magnets the primary residual gases are helium and hydrogen. The measured primary ionization rate for minimally ionizing protons in these two gases at atmospheric pressure is about 5.5 ion pairs $/ \mathrm{cm}$. For other beams, the cross section is proportional to $Z^{2}$ [5]. For the measurements reported here the bunch contained about $10^{8}$ ions which at the base pressure of $10^{-9}$ torr would have produced about 14 detected electrons on each bunch passage. To be able to measure single-bunch profiles the pressure in the detector chamber was raised to $10^{-7}$ torr by outgassing a $\mathrm{Ti}$ filament in the adjacent vacuum pump. We estimate the signal consisted of 3000-6000 electrons.

To obtain an accurate profile it is necessary for each electron to travel along a plane parallel to the beam axis and perpendicular to the detector anode. However, the ionizing events impart momenta to the electrons, and the electrons are released in the space-charge electric field of the beam. The impulse from the collision and the transverse component of the space-charge electric field can move the electrons perpendicular to the beam axis resulting in a measured profile which is wider than the beam. To counter these effects the detector is placed in a $0.14 \mathrm{~T}$ magnetic field oriented parallel to the electric field which sweeps the electrons from the beamline.

Consider the defocusing effect of the space-charge electric field of the beam bunch. This is a radial field whose strength increases with radius to a maximum near the beam s edge. At each point in the beamline the space-charge field has a component which is parallel to the desired drift direction and one that is perpendicular to it. Only the perpendicular component, $\mathrm{E}_{1}$, has a defocusing effect.

An electron subjected to perpendicular electric and magnetic fields will drift with a cycloidal motion in the direction defined by the vector product $\mathbf{E} \times \mathbf{B}$ with a velocity of, $v=(\mathbf{E} \times \mathbf{B}) / \mathrm{B}^{2}$, and a gyration radius of, $\mathrm{R}=\mathrm{Em} / \mathrm{qB}^{2}$. During bunch passage electrons respond to any transverse electric field by moving with a cycloidal pattern 
parallel to the collector anodes. The maximum space-charge field in RHIC is about $10^{5}$ $\mathrm{V} / \mathrm{m}$. With the magnetic field the gyration radius is less than $40 \mu \mathrm{m}$ everywhere in the beamline and electrons drift parallel to the anode by less than $0.2 \mathrm{~mm}$. Therefore profile broadening by the space-charge field is eliminated. Also, by the same mechanism, profile distortions from errors in the sweep electric field are eliminated.

The second defocusing effect is the momentum impulse from the ionizing collision. Over $95 \%$ of recoil electrons have energies less than $500 \mathrm{eV} \mathrm{[6].} \mathrm{In} \mathrm{a} \mathrm{magnetic} \mathrm{field} \mathrm{an}$ electron trajectory is a helix whose radius depends inversely on the transverse component of momentum. A $0.14 \mathrm{~T}$ field confines a $500 \mathrm{eV}$ electron to a Larmor radius of $0.54 \mathrm{~mm}$ which is less than the spacing between collector anodes. With the magnet most of the electrons are collected on the anodes over which they are formed.

\section{DETECTOR CONSTRUCTION}

Figure 1 is a cross section of the IPM transducer from the perspective of looking along the beamline. The detector vacuum chamber is made from a one-meter section of $10 \times 15 \mathrm{~cm}$ rectangular stainless steel tubing with a short piece of the same tubing welded into the side to form a $\mathrm{T}$. The detector assembly and all electrical feedthroughs are mounted on a 10 conflat-type flange which mounts on the side port. Two stainless steel brackets hold the collector anode and microchannel plate assembly on one side of the beam and a sweep electrode with electron suppression grid on the other side. The sweep field is generated by a $3 \mathrm{kV}$ supply which floats on top of the $\mathrm{MCP}$ bias voltage.

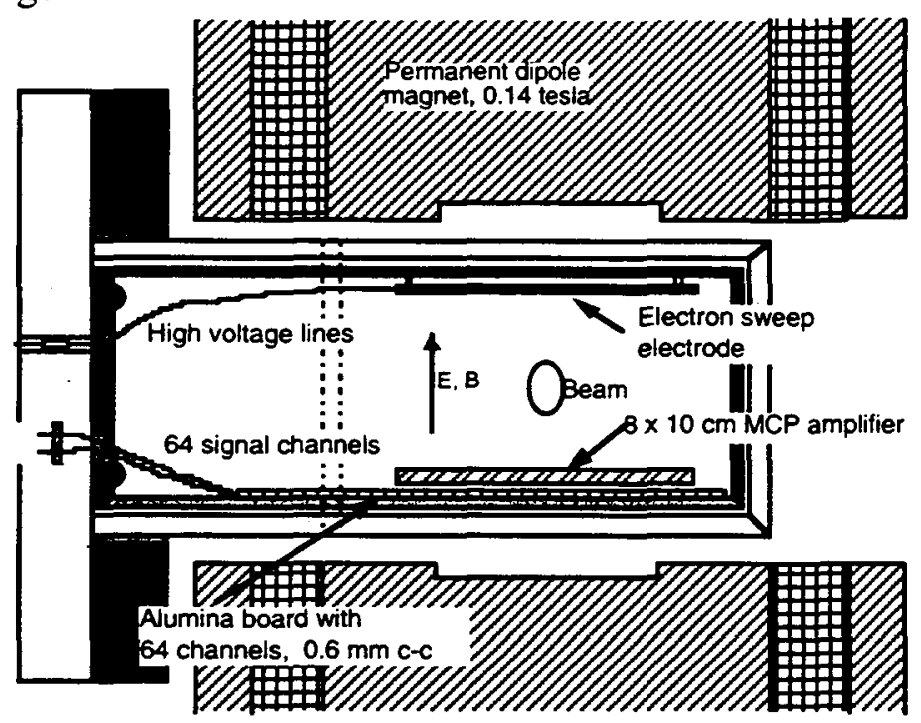

FIGURE 1. Cross sectional view of IPM detector head.

The detector chamber is mounted in the gaps of two 30-cm long C-shaped permanent dipole magnets separated by a $30 \mathrm{~cm}$ gap. One is centered on the detector to focus the 
electrons and the second corrects the beam deflection caused by the first. The magnet pole tips are shaped to produce parallel field lines within an area of $38 \mathrm{~mm}$ parallel to the collector by $63 \mathrm{~mm}$ perpendicular to it. Within this collection area the maximum field-line transverse runout is $0.034 \mathrm{~mm}$ which is $6 \%$ of a channel width. The collector board is alumina metallized with gold. A chevron $8 \times 10 \mathrm{~cm}$ microchannel plate detector $[7,8]$ is screwed directly to the board with a kapton gasket providing electrical insulation.

A bunch passage delivers charge pulses to the anodes. Each anode channel is connected to a charge-sensitive preamplifier which integrates the pulse and a shaper which produces an ouput pulse with a base width of $100 \mathrm{~ns}$. The preamplifier has a charge sensitivity of $0.6 \mathrm{~V} / \mathrm{pC}$ and a decay time constant of $20 \mathrm{~ns}$, and the shaper has a voltage gain of 10. The shaper output is amplified by a AD811 op amp [9] set to a gain of 2 , and coupled into a $100 \Omega$ balanced line via a pulse transformer. The preamps are in a chassis box mounted on the vacuum feedthrough to minimize signal lead length.

The individual-channel pulses are digitized by Hytec VTR2535 8-channel VME digitizers [10]. Each digitizer channel has a dedicated AD9220 12-bit, 10MSPS ADC backed by $128 \mathrm{k}$ words of memory. The digitizers are triggered by a timing module synchronized to the beam-sync clock [11]. During beam measurement the data are written into memory on the digitizer cards. Acquisition is then stopped and the data are read by the front end computer in the VME chassis and sorted into profiles.

\section{MEASUREMENTS}

During the commissioning experiments a data set was taken consisting of individual profiles of a single bunch on 2000 consecutive turns starting 25 turns after injection. We waited 25 turns because about $30 \%$ of the beam was being lost on the first few turns. The IPM $s$ are extremely sensitive to radiation so clean profiles require low beam loss.

Figure 2 shows a beam profile generated by a single pass of one bunch with a Gaussian curve fit to it. The ms width of the fitted curve is $\sigma=4.42 \mathrm{~mm}$ and the calculated betatron function at the IPM is $185 \mathrm{~m}$. On the RHIC project it is standard to use the normalized $95 \%$ emittance which is calculated from,

$$
\sigma=\sqrt{\frac{\varepsilon_{n} \beta(s)}{6 \pi(\beta \gamma)}}
$$

Here $\beta(s)$ is the betatron function and $(\beta \gamma)$ is the relativistic factor which was approximately 12 in these measurements. This formula gives a measured emittance of $7.6 \times 10^{-6} \mathrm{~m}$. The measured beam had about $10 \%$ of the design intensity and the design emittance is $10^{-5} \mathrm{~m}$. 


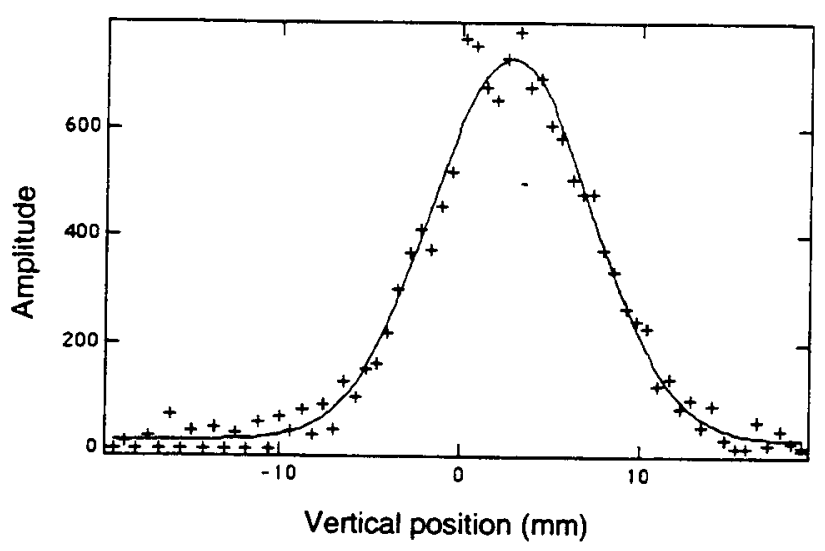

FIGURE 2. A measured profile from a single gold bunch containing $10^{8}$ ions. The markers are the detector data and the solid line is a Gaussian curve fit to the data.

Figure 3 is a mountain-range plot of the first 100 measured turns showing large betatron oscillations at injection. On turns 29 and 69 there was beam loss upstream of the detector resulting in a large fraction of the MCP width being excited by radiation. The centers of the first 128 profiles were found and a Fourier transform on these data gave a fractional tune of 0.18 , which was the tune measured with the BPM $\mathrm{s}$.

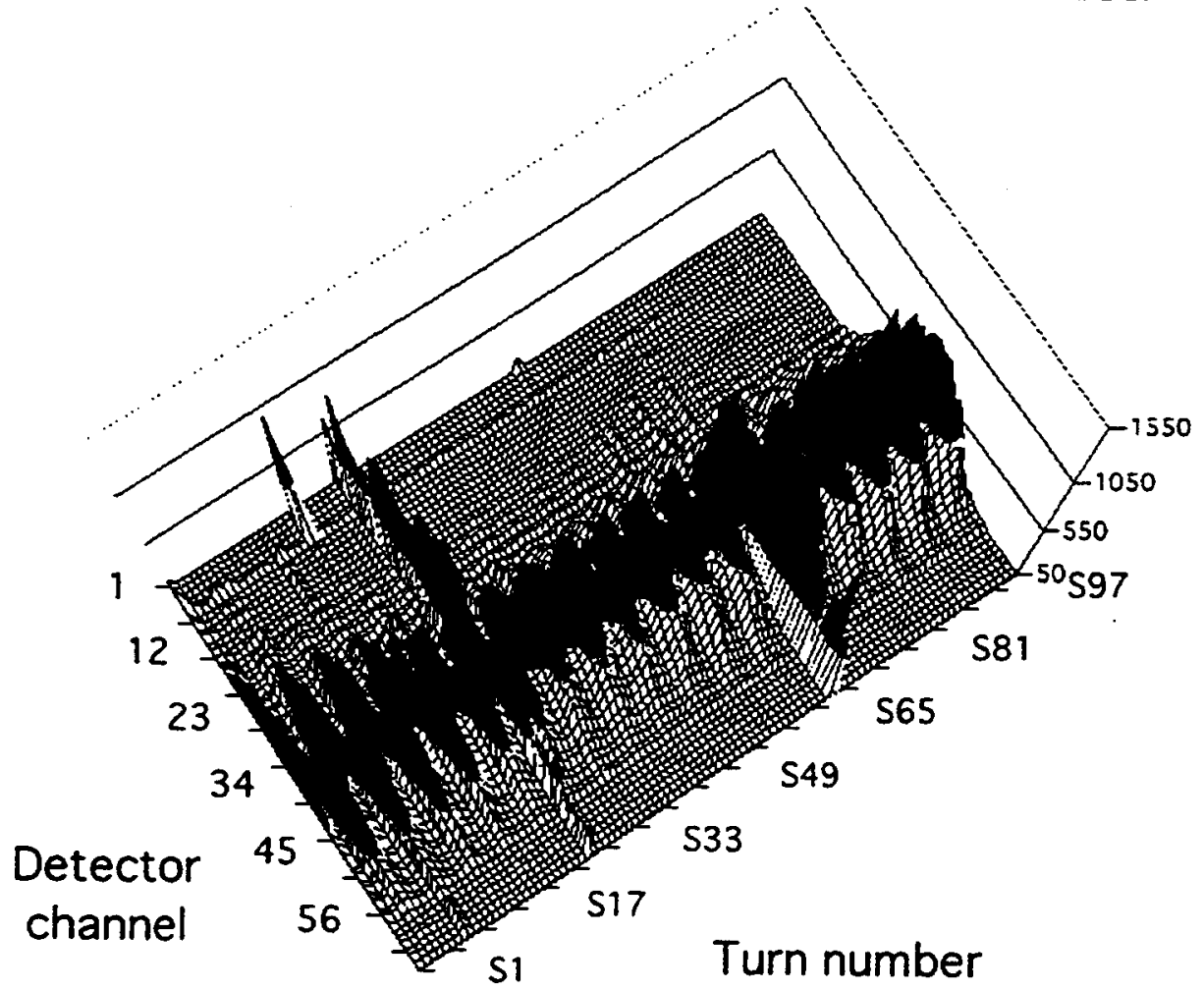

FIGURE 3. A mountain-range plot of 100 transverse profiles of a single bunch on consecutive turns. The first profile is on the left. The profile shown in fig. 2 is number 40 on this plot. 


\section{CONCLUSION}

The transverse profile of a single beam bunch was measured on each of 2000 turns during the commissioning of RHIC. A Gaussian fit to a single profile gives a beam emittance which agrees with the beam emittance in the AGS accelerator which injects beam into RHIC [12]. Several consecutive profiles are assembled into a mountainrange plot which shows betatron oscillations at injection.

\section{ACKNOWLEDGEMENTS}

The IPM was built with help from Peter Cameron, John Cupolo, Veljko Radeka, William Ryan, Robert Sikora, Dmitri Stephani, and Nickolaos Tsoupas all at Brookhaven National Lab and Lee Woodworth and Dave Wilkie from Raytheon Electronic Systems Division in Sudbury, MA. This work was funded by the Department of Energy.

\section{REFERENCES}

1. http://www.rhichome.bnl.gov/RHIC/index.html

2. R. Connolly, P. Cameron, W. Ryan, T.J. Shea, R. Sikora, and N. Tsoupas, A Prototype Ionization Profile Monitor for RHIC, Proceedings of the 1997 IEEE Particle Accelerator Conference, pg. 2152.

3. R. Connolly, P. Cameron, R. Connolly, R. Michnoff, V. Radeka, W. Ryan, T. Shea, R. Sikora, D. Stephani, S. Tepikian, and N. Tsoupas, The RHIC Ionization Beam Profile Monitor, Proceedings of the 1999 IEEE Particle Accelerator Conference.

4. M. Conte and W. MacKay, An Introduction to the Physics of Particle Accelerators, World Scientific, River Edge, NJ, 1994.

5. F. Sauli, Principles of Operation of Multiwire Proportional and Drift Chambers, CERN 77-09 (1977).

6. J. D. Jackson, Classical Electrodynamics, p. 430, John Wiley and Sons, New York, 1962.

7. J. Wiza, Microchannel Plate Detectors, Nucl. Instr. Meth. 162 (1979) 587-601.

8. Galileo Electro-Optics Corp., Sturbridge, MA 01566.

9. Analog Devices, Norwood, MA 02062-9106.

10. Hytec Electronics Ltd., Reading, Berks. RG20JT, UK.

11. H. Hartmann and T. Kerner, RHIC Beam Synchronous Trigger Module, Proceedings of the 1999 IEEE Particle Accelerator Conference.

12. T. Roser, private communication. 